\title{
Evaluating the Potential Benefit of Autostereoscopy in Laparoscopic Sacrocolpopexy through VR Simulation*
}

\author{
Jef De Smet ${ }^{1}$, Vladimir Poliakov ${ }^{1}$, Kenan Niu ${ }^{1}$, Frédérique Chesterman ${ }^{2}$, Johan Fornier ${ }^{2}$, \\ Mirza Awais Ahmad ${ }^{1}$, Mouloud Ourak ${ }^{1}$, Viktor Vörös ${ }^{1}$, Jan Deprest ${ }^{3,4}$ and Emmanuel Vander Poorten ${ }^{1}$
}

\begin{abstract}
During laparoscopic sacrocolpopexy, pelvic organ prolapse is repaired by suturing one side of a synthetic mesh around the vaginal vault while stapling the other end to the sacrum, restoring the anatomical position of the vagina. A perineal assistant positions and tensions the vault with a vaginal manipulator instrument to properly expose the vaginal tissue to the laparoscopic surgeon. A technical difficulty during this surgery is the loss of depth perception due to visualization of the patient's internals on a 2D screen. Especially during precise surgical tasks, a more natural way to understand the distance between the laparoscopic instruments and the surgical region of interest could be advantageous. This work describes an exploratory study to investigate the potential of introducing 3D visualization into this surgical intervention. More in particular, experimentation is conducted with autostereoscopic display technology. A mixed reality setup was constructed featuring a virtual reality model of the vagina, $2 \mathrm{D}$ and $3 \mathrm{D}$ visualization, a physical interface representing the tissue of the body wall and a tracking system to track instrument motion. An experiment was conducted whereby the participants had to navigate the instrument to a number of pre-defined locations under $2 \mathrm{D}$ or $3 \mathrm{D}$ visualization. Compared to $2 \mathrm{D}$, a considerable reduction in average task time $(-42.9 \%)$, travelled path lenght $(-31.8 \%)$ and errors $(-52.2 \%)$ was observed when performing the experiment in 3D. Where this work demonstrated a potential benefit of autostereoscopic visualization with respect to $2 \mathrm{D}$ visualization, in future work we wish to investigate if there also exists a benefit when comparing this technology with conventional stereoscopic visualization and whether stereoscopy can be used for (semi-) automated guidance during robotic laparoscopy.
\end{abstract}

\section{INTRODUCTION}

Minimally invasive surgery (MIS) is taking in an increasingly important role in modern surgery [1]. The main benefits of MIS over open surgery are reduced blood loss, lower morbidity, a shorter recovery-time, less post-operative pain and better cosmetic outcomes for the patient [2], [3], [4]. These benefits arise from the use of long, slender instruments that are inserted through small incisions of approximately $1 \mathrm{~cm}$ in the patient's abdominal wall.

One procedure to execute in a minimally invasive fashion, is treatment of pelvic organ prolapse (POP). POP is a medical condition whereby the supporting muscles and ligaments of the pelvic organs (small bowels, rectum, bladder, uterus and

\footnotetext{
*This work was supported by Barco NV (Kortrijk, Belgium).

${ }^{1} \mathrm{KU}$ Leuven, Department of Mechanical Engineering, Celestijnenlaan 300, Leuven, Belgium jef . desmet a kuleuven. be

${ }^{2}$ Barco NV, Healthcare Division, Beneluxpark 21, Kortrijk, Belgium

${ }^{3}$ KU Leuven, Department of Development and Regeneration, Herestraat 49, Leuven, Belgium

${ }^{4}$ UZ Leuven, Department of Obstetrics and Gynaecology, Herestraat 49, Leuven, Belgium
}

vagina) are defective. As a consequence, the pelvic organs move away from their anatomical position and descent into or outside the vaginal canal, causing prolapse [5]. The main risk factors for developing POP are vaginal childbirth, aging, an increased body mass index (BMI) and hysterectomy [6]. It is estimated that 2 out of 3 of parous women have anatomical evidence of POP [7].

During laparoscopic sacrocolpopexy (LSCP), POP is repaired by fixating the vaginal vault to the sacrum with a synthetic graft/mesh restoring its anatomical position and preserving vaginal function [8]. Due to the reduced patient trauma, it became increasingly popular in the last decade [9], [10]. At least three people are present around the operation table: a surgeon and an assistant that operate the laparoscopic instruments through four or five cannulas in the abdominal wall and a second perineal assistant who sits between the legs of the patient and uses a vaginal manipulator to precisely position and tension the vaginal vault.

Unfortunately, many technical challenges arise while performing LSCP. Associated with MIS, instrument motions are constrained to rotate around the incision: no direct access to organs is possible, haptic feedback is reduced and handeye coordination is complex [1]. Most often, the patient's internals are displayed on a $2 \mathrm{D}$ screen, leading to a loss of depth perception which further complicates performing fine tasks such as suturing [11]. Long operation times - 224 minutes on average [12] — and non-ergonomic postures lead to discomfort in neck and upper extremities and high stress levels [13], [14]. As much as $74 \%$ of laparoscopic surgeons reported physical complaints [15]. Overall there is a steep learning curve [16] of at least 60 interventions [17].

This work evaluates the potential benefits of $3 \mathrm{D}$ vision as a means of reintroducing depth perception to laparoscopic surgery; and more specific to LSCP.

The paper is built up as follows: Firstly, section [I] describes the state of the art w.r.t. stereoscopic imaging and stresses where the current study fills the gap in literature. Secondly, section III explains the experimental setup and the quantitative metrics used to compare $2 \mathrm{D}$ and $3 \mathrm{D}$ in a LSCP virtual reality (VR) environment. Section IV analyses the outcomes of the experiment and interpretes the results. Lastly, section $\mathrm{V}$ makes conclusions on the study results and mentions future work.

\section{STATE OF THE ART}

Since the second half of 1990s stereoscopic imaging has been receiving a lot of attention in the medical domain 
as a means of reintroducing depth perception. In the past, a few researches have investigated the potential benefits of using three-dimensional vision in laparoscopic training compared to a conventional two-dimensional camera. Several works confirm the hypothesis that using a stereoscopic camera contributes to depth perception of a surgeon and improves overall efficiency [11], [18], [19], [20], although some researches reported a negative impact of introducing a 3D camera to the trainees [21], [22]. It is believed that these negative results originated from the early stage of the technology at that time. Since these early days technology has evolved significantly.

Other studies compare the rate of skill acquisition using 2D and 3D laparoscopy [23], [24]. Here, subjects were asked to perform a series of exercises using a conventional twodimensional endoscope and a stereoscope for visualization. Romero-Loera et al. reports improvement of performance and precision when using the stereoscopic camera [23]. Özsoy et al. indicates that, regardless of imaging modality, previous task experience has an important impact on performance. Nonetheless, 3D laparoscopy seemed to facilitate the learning curve for novice surgeons [24].

Guana et al. conducted a comparative analysis of skill transfer in pediatric surgery simulation using threedimensional and two-dimensional high-definition endoscopic cameras [19]. Similarly, participants needed less time to finish the exercise, and at the same time most of participants have found 3D laparoscopy easier to perform overall (65\%). Nevertheless, this technology has been associated with some difficulties: $25 \%$ of participants experienced headache during the session and nausea occurred for $20 \%$ of participants. One possible explanation for these issues is the fact that usage of polarized glasses decreased the level of brightness, thus increasing the fatigue level imposed on the eyes of the user.

A cross-sectional comparison was performed by Blavier et al. [11] to effectively evaluate the perceptual impact of twodimensional versus three-dimensional view in laparoscopic and robot-assisted surgery on the learning curve. Each participant performed a series of exercises using a robotic surgical system and conventional laparoscopy, both with 2D and 3D cameras. The work describes a 'perspective switch' in which the user switches to a different view perspective, from $2 \mathrm{D}$ to $3 \mathrm{D}$ or the opposite way. As a result, a significant positive shift of efficiency has been detected when switching from $2 \mathrm{D}$ to $3 \mathrm{D}$ view. Contrarily, switching from $3 \mathrm{D}$ to $2 \mathrm{D}$ led to decline in performance of the subject. Subsequently, two main conclusions can be drawn from this study: first, 3D viewing technology offers better depth perception compared to $2 \mathrm{D}$; and second, stereoscopic view is not fully suitable for training in conventional 2D laparoscopy as it builds up certain expectations for the user.

As for potential benefits of using autostereoscopy or glasses-free 3D in surgery, the topic is yet to be investigated as the technology remains in early development stage, and very few devices are available on the market. Several authors [25], [26], [27] advocate glasses-free technology as the next evolutionary step of $3 \mathrm{D}$, enabling the same level of depth

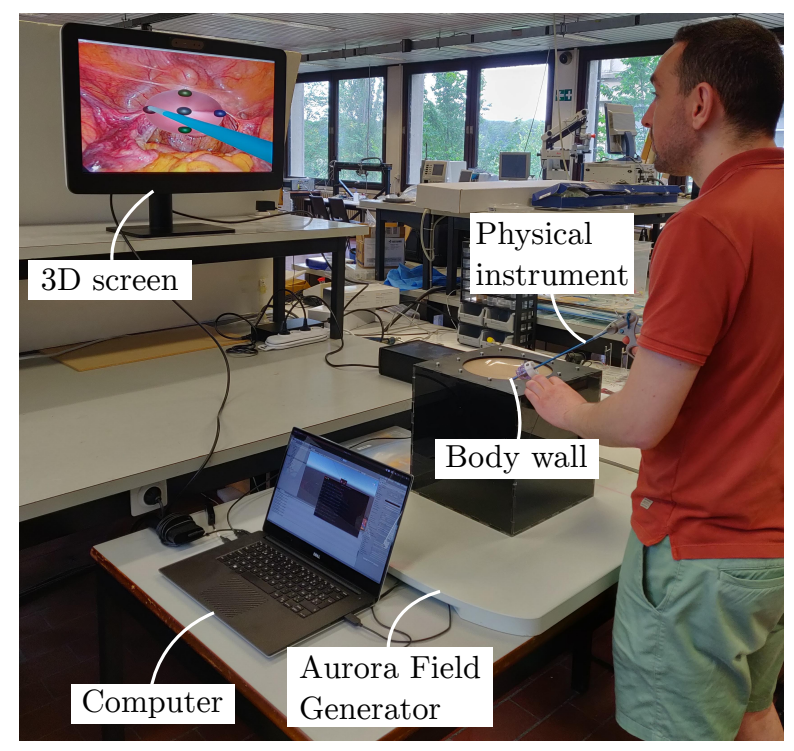

Fig. 1. Experimental setup: participants were asked to manipulate a physical instrument which was projected in the VR world to touch a series of markers on the vaginal vault. A box trainer containing a synthetic body wall was used to mechanically constrain the instrument's movement around a pivot point.

perception and at the same time decreasing the effect of fatigue imposed on the user. Particularly in the medical field, a few case studies reported successful interventions using glasses-free 3D display for visualization. Jang et al. reported successful thoratcoscopic thymectomy using glasses-free 3D technology [28]; Zeng et al. reported successful trans-oral thyroidectomy using glasses-free $3 \mathrm{D}$ visualization equipment [26]; Li described the case of radical resection for lung cancer using 3D-thoracosope and glasses-free 3D screen [29].

As far as the authors are aware of, no research has been conducted to quantatively evaluate the benefit of glass-free 3D in gynaecological laparoscopy. Moreover, none of the current studies focused on using this technology in LSCP nor on the potential of this technology in surgical training for LSCP. Hence, the current study attempts to close this gap by investigating potential benefits of using autostereoscopy in a VR training system for LSCP and comparing it to conventional two-dimensional laparoscopy. Future work will investigate whether a benefit exists with conventional stereoscopy.

\section{METHODS}

To compare 2D and 3D, a VR environment has been developed in Unity (Unity Technologies, San Francisco, USA) mimicking the view on the vaginal vault during LSCP. A physical instrument (DetachaTip ${ }^{\mathrm{R}}$ Scissors, CONMED, New York, USA) is inserted through a $5 \mathrm{~mm}$ diameter trocar in a box trainer containing a $8 \mathrm{~mm}$ thick synthetic body wall (Ecoflex $^{\mathrm{TM}}$, Smooth-On, Inc., Pennsylvania, USA) duplicating keyhole interaction. To transform the pose of the physical instrument to a virtual instrument, an electromagnetic field generator (Aurora System, Northern Digital Inc., Canada) is 
placed below the box trainer and a tracking probe is attached to the instrument handle. The 3D screen is placed $1.7 \mathrm{~m}$ in front of the participant at eye level, similar to a real LSCPscenario. The setup is shown on Fig. 1 .

Some intermediate steps are necessary to communicate the pose of the physical instrument to the virtual one in Unity: A Linux computer running Robot Operating System (ROS) middleware reads the electromagnetic field generator (at $40 \mathrm{~Hz}$ ) from a USB-connection. The recorded data, i.e. the pose of the physical instrument $i_{p}$-consisting out of a Cartestian position ${ }_{i}^{w} \boldsymbol{p}=\left[\begin{array}{lll}p_{x} & p_{y} & p_{z}\end{array}\right]^{T}$ and a quaternion ${ }_{i_{p}}^{w} \boldsymbol{q}=\left[\begin{array}{llll}q_{w} & q_{x} & q_{y} & q_{z}\end{array}\right]^{T}$-is then published as a ROS topic and sent to an internal network socket read by Unity. ROS\# (Siemens AG, Munich, Germany), a set of open source software libraries, is used for this communication step.

To obtain the pose of the virtual instrument $i_{v}$ in the Unity frame $\{\boldsymbol{u}\}$, the pose of the physical instrument w.r.t. the origin of the electromagnetic field generator — which acts as the origin of physical world frame $\{\boldsymbol{w}\}$-is converted to a transformation matrix ${ }_{i_{p}}^{w} \boldsymbol{T}$ and imposed on the virtual instrument in Unity. More precise, ${ }_{i}^{w} \boldsymbol{q}$ is transformed to a rotation matrix ${ }_{i_{p}}^{w} \boldsymbol{R}$

$$
{ }_{i_{p}}^{w} \boldsymbol{R}=\left[\begin{array}{ccc}
1-2\left(q_{y}^{2}+q_{z}^{2}\right) & 2\left(q_{x} q_{y}-q_{w} q_{z}\right) & 2\left(q_{w} q_{y}+q_{x} q_{z}\right) \\
2\left(q_{x} q_{y}+q_{w} q_{z}\right) & 1-2\left(q_{x}^{2}+q_{z}^{2}\right) & 2\left(q_{y} q_{z}-q_{w} q_{x}\right) \\
2\left(q_{x} q_{z}-q_{w} q_{y}\right) & 2\left(q_{w} q_{x}+q_{y} q_{z}\right) & 1-2\left(q_{x}^{2}+q_{y}^{2}\right)
\end{array}\right]
$$

which is used to find the pose of the physical instrument

$$
{ }_{i_{p}}^{w} \boldsymbol{T}=\left[\begin{array}{c|c}
{ }_{i_{p}}^{w} \boldsymbol{R} & i_{p}^{w} \boldsymbol{p} \\
\hline 0_{1 \times 3} & 1
\end{array}\right],
$$

and

$$
{ }_{i_{v}}^{u} \boldsymbol{T}={ }_{i_{p}}^{w} \boldsymbol{T} .
$$

With ${ }_{i_{v}}^{w} \boldsymbol{T}$ the transformation matrix of the virtual instrument in the Unity frame.

Lastly, the Unity environment is displayed on an autostereoscopic screen from Barco NV (Kortrijk, Belgium). This screen consists of a high-quality liquid crystal display (LCD) with image diagonal of 27 " and a high-resolution of $3840 \times 2160$ pixels $(4 \mathrm{~K})$. The refresh rate of the LCD is $60 \mathrm{~Hz}$. The camera embedded in the screen tracks the position of the eyes of the user in front of the screen and the images are rendered in such a way that the 3D stereoscopic image will be observed by the user being tracked. Fig. 2 shows the data flow diagram.

\section{A. Simulated Task and Protocol}

A task has been designed in which participants (sample size $n=10$ ) had to manipulate the physical instrument which was projected in the VR environment. To evaluate depth perception, users had to touch a series of five spherical markers on the vaginal vault which where placed at different locations and depths w.r.t. the camera. Each marker, the vagina and the virtual instrument are equipped with a 'primitive collider type' (spere- and capsule colliders) built into Unity. Primitive colliders avoid high computational costs - when compared to a mesh collider - and are accurate enough for the simple object shapes. A marker is activated

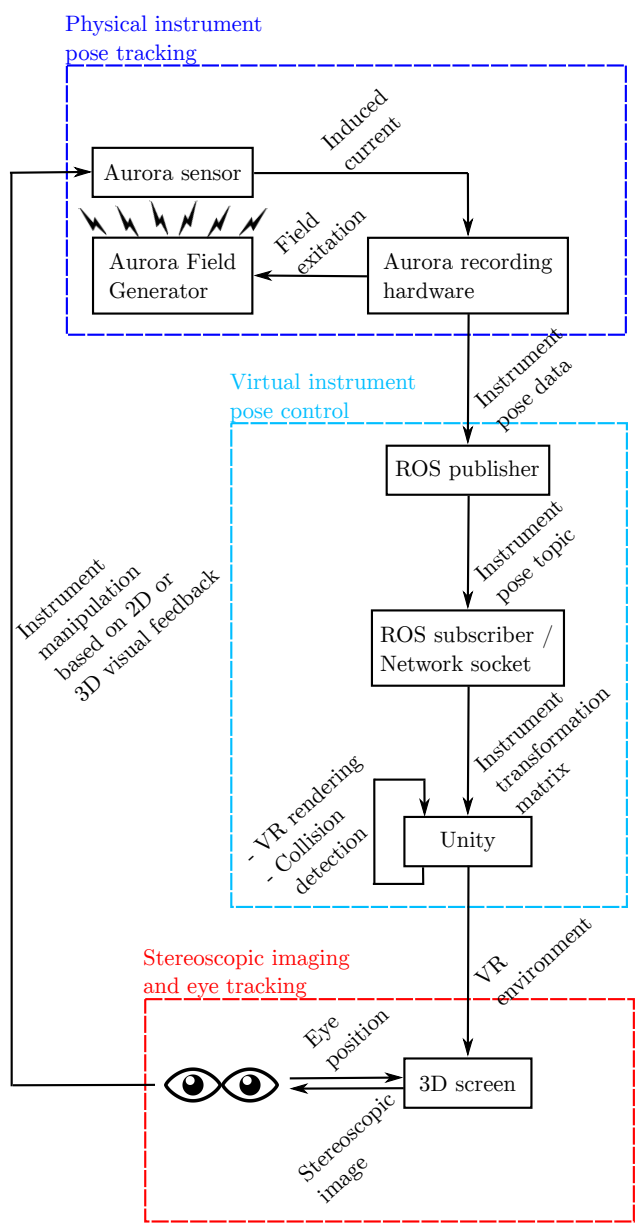

Fig. 2. Data flow diagram of the autostereoscopic LSCP simulation.

TABLE I

DIMENSIONS OF THE SIMULATED OBJECTS.

\begin{tabular}{ll}
\hline Object & Dimensions $[\mathrm{mm}]$ \\
\hline Vagina & $200 \times 60($ length $\times \varnothing)$ \\
Spherical markers & $10(\varnothing)$ \\
Instrument & $330 \times 5($ length $\times \varnothing)$ \\
\hline
\end{tabular}

when a collision with the instrument tip is detected. To give visual feedback activated markers are lit up whereas deactivated ones stay dark, as shown on Fig. 3. The dimensions of the simulated objects are summarized in Table $\mathrm{I}$

In order to better evaluate depth perception, the task was made more challenging by deactivating all markers when a collision between the vagina and the instrument tip was detected. The user would then also get a penalty point or error. This way, participants had to make sure to not to overshoot when approaching a marker. Only when all five markers are simultaniously activated, the vaginal vault moves to a new position as if the perineal assistant would reposition the vaginal manipulator instrument. A total of four vaginal positions or phases were included in the task as shown on 


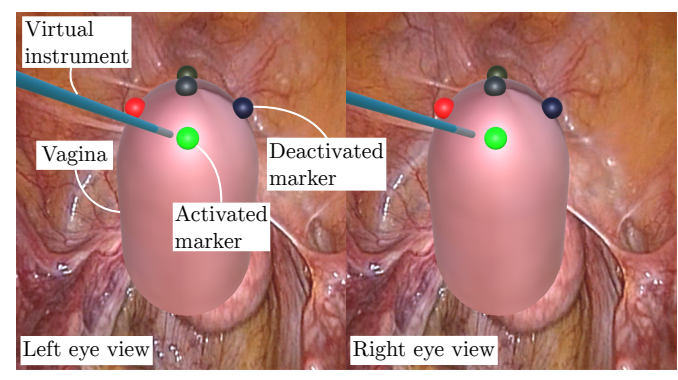

Fig. 3. Stereostopic image displaying the view on the vaginal vault during LSCP. A series of five markers is attached to the vagina as a means to evaluate depth perception. A marker is activated when a collision with the virtual instrument is detected. All markers are deactivated when a collision between the virtual instrument and the vagina is detected.

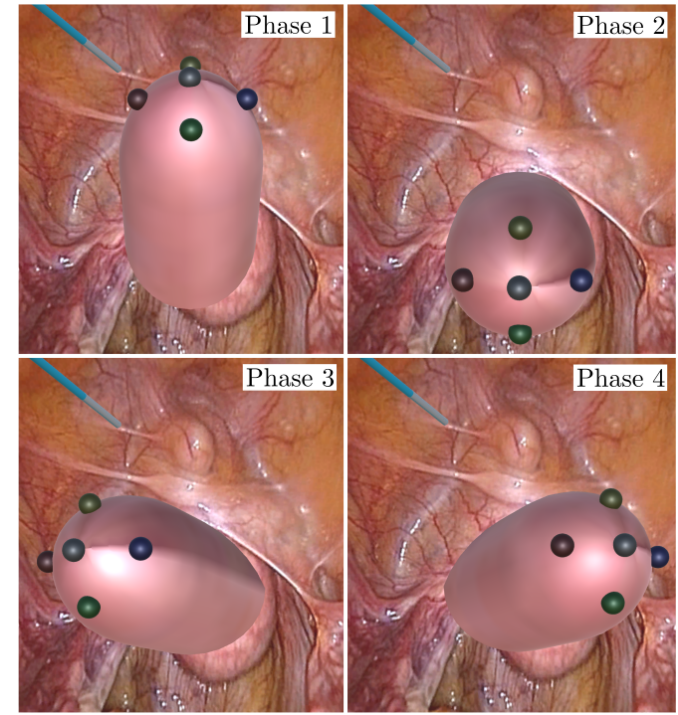

Fig. 4. Different phases during the task. The vagina moves to a next phase only when all five markers are active.

Fig. 4. The angle between the camera and vaginal center axes are displayed in Table III

Every participant had to complete the task in $2 \mathrm{D}$ and $3 \mathrm{D}$. Switching the VR scene between 2D and 3D was done by giving zero-distance between the stereoscopic camera images which results in a $2 \mathrm{D}$ projection on the stereoscopic screen. To avoid bias due to training, half of the participants did the 2D experiment first while the other half started with 3D. Each participant got the chance to complete phase 1 of the task before starting the experiment to get accustomed to the instrument movement. Several parameters are recorded:

- the time $t$ it takes to complete the task;

- the time to complete an individual phase $t_{i}$;

- the number of errors made when touching the vaginal tissue, in total $\epsilon$;

- and for each stage $\epsilon_{i}$;

- the travelled distance of the instrument tip $d$.

To compare the results and search for statistically significant differences between 2D and 3D, a Mann-WhitneyWilcoxon (MWW) test was performed using using Math-
TABLE II

ANGLES OF THE VAGINA'S CENTER AXIS W.R.T. THE CAMERA'S CENTER AXIS DURING DIFFERENT PHASES.

\begin{tabular}{ll}
\hline Phase & Angle $\left[^{\circ}\right]$ \\
\hline 1 & +45 (up) \\
2 & -10 (down) \\
3 & +45 (left) \\
4 & -45 (right) \\
\hline
\end{tabular}

ematica (Wolfram Mathematica 10.3.0, Illinois, USA). A difference between one of the abovementioned parameters is considered significant when the obtained $p$-value is lower than 0.05 .

\section{RESULTS AND DISCUSSION}

Table III gives an overview of the time, number of errors and travelled distance of the instrument tip for both 2D and $3 \mathrm{D}$ experiments. The average values $\bar{x}$ and the standard deviation $\sigma$ are calculated separately for the 2D- and 3D case. In the bottom row of the table $p$-values are displayed and denoted with a ${ }^{*}$ ' when significant $(<0.05)$.

When using 3D, the average participant scores better on task time $(171.9 \mathrm{~s}$ vs. $301.0 \mathrm{~s})$, total number of errors (4.4 vs. 9.2) and travelled distance ( $3550.0 \mathrm{~mm}$ vs. $5207.6 \mathrm{~mm}$ ). Only participant 8 tends to perform better in the 2D case. Although these differences are small when compared to the other participants, indicating a reduced effect of the imaging technique on the task at hand for that person. Overall, compared to $2 \mathrm{D}$, a considerable reduction in task time $(-42.9 \%)$, travelled path lenght $(-31.8 \%)$ and errors $(-52.2 \%)$ is observed when performing the experiment in $3 \mathrm{D}$. The improvements in time $t$ to complete the task $(p=0.0022)$ and travelled distance $d(p=0.0376)$ when using $3 \mathrm{D}$ are significant. However, while there is a clear improvement in average total errors $\epsilon$ the difference is not statistically significant $(p=0.0578)$. This may be caused by the limited sample size $n$. Furthermore it must be said that the participants were not clinicians and had no to little experience with keyhole surgery. Additional research is needed to evaluate whether novices with a clinical background or expert surgeons would equally benefit from autostereoscopic vision as the population that was tested here.

Fig. 5 shows the workspace for the 2D and 3D experiment (participant 2). It is visible that when using 2D the participant tends to retract the instrument further backwards after touching a marker leading to an increased $d$. This is further confirmed by participant 9 expressing to be more confident when moving close to the vagina in 3D. Except from subject 7 and 8, other participants also show this behaviour.

\section{CONCLUSIONS}

An exploratory study was performed to investigate the potential of introducing 3D visualization into LSCP. More 
TABLE III

Overview of 2D AND 3D Results. SignificANT $p$-VAlues $(<0.05)$ ARE DENOtEd With A ${ }^{\prime}$ '.

\begin{tabular}{|c|c|c|c|c|c|c|c|c|c|c|c|c|}
\hline \multirow[b]{2}{*}{ Participant } & \multirow[b]{2}{*}{$2 \mathrm{D} / 3 \mathrm{D}$} & \multicolumn{5}{|c|}{ Time $[\mathrm{s}]$} & \multicolumn{5}{|c|}{ Errors } & \multirow[b]{2}{*}{$d[\mathrm{~mm}]$} \\
\hline & & $t_{1}$ & $t_{2}$ & $t_{3}$ & $t_{4}$ & $t$ & $\epsilon_{1}$ & $\epsilon_{2}$ & $\epsilon_{3}$ & $\epsilon_{4}$ & $\epsilon$ & \\
\hline \multirow[t]{2}{*}{1} & $2 \mathrm{D}$ & 62.4 & 84.5 & 52.0 & 89.6 & 288.5 & 2 & 3 & 2 & 3 & 10 & 5965.1 \\
\hline & $3 \mathrm{D}$ & 40.5 & 83.9 & 26.0 & 44.6 & 195.0 & 0 & 4 & 0 & 0 & 4 & 4303.8 \\
\hline \multirow[t]{2}{*}{2} & $2 \mathrm{D}$ & 115.9 & 111.8 & 70.5 & 109.9 & 408.1 & 1 & 2 & 0 & 0 & 3 & 7528.6 \\
\hline & $3 \mathrm{D}$ & 48.2 & 39.1 & 27.3 & 39.1 & 153.6 & 0 & 1 & 0 & 2 & 3 & 3413.1 \\
\hline \multirow[t]{2}{*}{3} & $2 \mathrm{D}$ & 66.2 & 103.6 & 35.1 & 69.6 & 274.5 & 2 & 7 & 0 & 4 & 13 & 4118.8 \\
\hline & $3 \mathrm{D}$ & 41.2 & 58.6 & 33.0 & 40.0 & 172.8 & 0 & 2 & 0 & 1 & 3 & 2818.7 \\
\hline \multirow[t]{2}{*}{4} & $2 \mathrm{D}$ & 58.9 & 35.7 & 91.1 & 60.6 & 246.3 & 4 & 2 & 4 & 3 & 13 & 4548.9 \\
\hline & $3 \mathrm{D}$ & 46.1 & 33.3 & 15.6 & 23.3 & 118.2 & 2 & 1 & 0 & 1 & 4 & 3398.2 \\
\hline \multirow[t]{2}{*}{5} & $2 \mathrm{D}$ & 72.4 & 51.4 & 43.8 & 57.6 & 225.2 & 1 & 0 & 2 & 4 & 7 & 4118.5 \\
\hline & $3 \mathrm{D}$ & 42.3 & 24.0 & 56.1 & 37.5 & 159.9 & 0 & 0 & 3 & 0 & 3 & 3189.6 \\
\hline \multirow[t]{2}{*}{6} & $2 \mathrm{D}$ & 52.9 & 97.3 & 212.0 & 84.3 & 445.5 & 1 & 4 & 11 & 1 & 17 & 7348.0 \\
\hline & $3 \mathrm{D}$ & 35.5 & 53.8 & 20.8 & 30.7 & 140.8 & 0 & 2 & 0 & 1 & 3 & 2979.3 \\
\hline \multirow[t]{2}{*}{7} & $2 \mathrm{D}$ & 53.3 & 35.9 & 36.9 & 58.2 & 184.2 & 1 & 1 & 0 & 1 & 3 & 3218.9 \\
\hline & $3 \mathrm{D}$ & 42.2 & 25.8 & 26.0 & 30.3 & 124.3 & 0 & 0 & 0 & 0 & 0 & 3255.5 \\
\hline \multirow[t]{2}{*}{8} & $2 \mathrm{D}$ & 57.0 & 32.8 & 101.0 & 126.3 & 317.0 & 3 & 1 & 7 & 2 & 13 & 5030.2 \\
\hline & $3 \mathrm{D}$ & 71.7 & 56.6 & 115.0 & 57.0 & 300.4 & 6 & 3 & 5 & 2 & 16 & 5284.9 \\
\hline \multirow[t]{2}{*}{9} & $2 \mathrm{D}$ & 39.9 & 76.9 & 47.6 & 33.5 & 197.9 & 0 & 3 & 0 & 0 & 3 & 3001.4 \\
\hline & $3 \mathrm{D}$ & 31.0 & 39.6 & 25.4 & 33.9 & 129.9 & 0 & 0 & 0 & 0 & 0 & 2293.4 \\
\hline \multirow{2}{*}{10} & $2 \mathrm{D}$ & 99.0 & 71.1 & 150.6 & 101.4 & 422.1 & 0 & 4 & 5 & 1 & 10 & 7197.7 \\
\hline & $3 \mathrm{D}$ & 45.0 & 43.5 & 95.8 & 39.3 & 223.7 & 0 & 2 & 5 & 1 & 8 & 4563.3 \\
\hline \multirow[t]{2}{*}{$\bar{x}$} & $2 \mathrm{D}$ & 67.8 & 70.1 & 84.0 & 79.1 & 301.0 & 1.5 & 2.7 & 3.1 & 1.9 & 9.2 & 5207.6 \\
\hline & $3 \mathrm{D}$ & 44.4 & 45.8 & 44.1 & 37.6 & 171.9 & 0.8 & 1.5 & 1.3 & 0.8 & 4.4 & 3550.0 \\
\hline \multirow[t]{2}{*}{$\sigma$} & $2 \mathrm{D}$ & 23.0 & 29.8 & 57.7 & 28.3 & 95.1 & 1.3 & 2.0 & 3.7 & 1.5 & 5.0 & 1704.9 \\
\hline & $3 \mathrm{D}$ & 10.9 & 18.0 & 34.3 & 9.2 & 55.8 & 1.9 & 1.4 & 2.2 & 0.8 & 4.6 & 900.8 \\
\hline$p$ & & $0.0073 *$ & 0.1041 & $0.0257 *$ & $0.0013^{*}$ & $0.0022^{*}$ & $0.0465^{*}$ & 0.1658 & 0.2292 & 0.1075 & 0.0578 & $0.0376^{*}$ \\
\hline
\end{tabular}
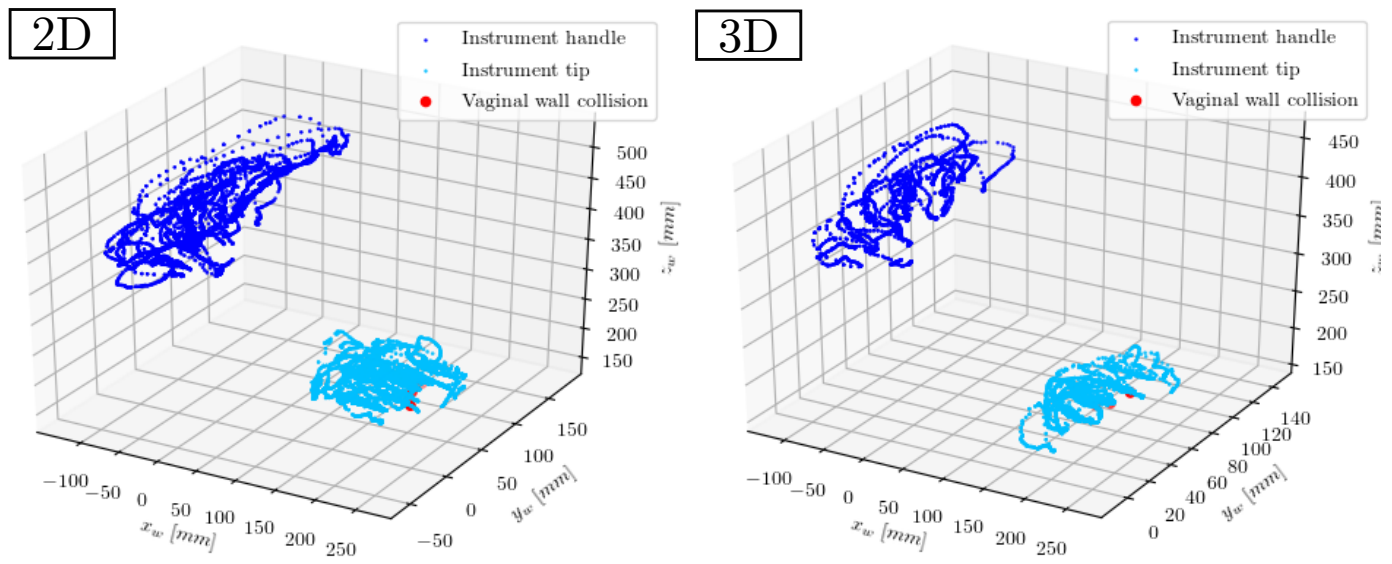

Fig. 5. Comparison of the instrument workspace when performing the experiment (participant 2). In the 2D-case the participant tends to retract the instrument further backwards after touching a marker.

specific, an experimental setup was designed and implemented to be able to test the effect of autostereoscopic display technology. The experiment was tailored to evaluate the difference in depth perception between 2D and 3D imaging. The used metrics to evaluate depth perception are time to complete the task $t$, the amount of errors $\epsilon$ when touching the wrong tissue and the travelled distance $d$ of the instrument tip. On average, all metrics show a decrease when going from $2 \mathrm{D}$ - to 3D vision. However, only the decrease in $t$ and $d$ is statistically significant. Participant 8 is the only one showing improved results when using $2 \mathrm{D}$, but the differences are small compared to the other's indicating a lower correlation between the used imaging technique and task performance. When plotting the instrument workspace it became clear that the participants tend to retract the instrument further away from the vaginal vault to avoid making erroneous contact.

Overall, 3D vision seems to facilitate depth perception for LSCP. Future work on this topic might include but is 
not limited to: adding haptic feedback towards the user to enable feeling tissue contact forces. This might influence the task as the participant doesn't need to rely only on vision anymore. A way to add haptic feedback can be to create a synthetic model of the vaginal vault or to perform in-vivo tests. Nonetheless, for evaluating depth perception it seems rather fair to limit the experiment to only visual feedback for the user. The sample size of the experiment was limited to only 10 non-expert subjects having no experience in performing laparoscopy. It would be interesting how experienced laparoscopists perform given they are used to force feedback while performing surgery. Also a lot of laparoscopists are used to $2 \mathrm{D}$ visualization and thus they might perform better under these circumstances. Similarly to this study, it seems also interesting to investigate whether there also exists a benefit when comparing this technology with conventional stereoscopic visualization for laparoscopic surgery. Lastly, we wish to investigate how 3D stereoscopy can be used for (semi-) automated guidance for robotic laparoscopic surgery.

\section{ACKNOWLEDGMENT}

The researchers would like to thank Barco NV for providing KU Leuven a 3D screen prototype for research purposes.

\section{REFERENCES}

[1] G. G. Hamad and M. Curet, "Minimally invasive surgery," The American Journal of Surgery, vol. 199, pp. 263-265, feb 2010.

[2] A.-L. W. M. Coolen, A. M. J. van Oudheusden, B. W. J. Mol, H. W. F. van Eijndhoven, J.-P. W. R. Roovers, and M. Y. Bongers, "Laparoscopic sacrocolpopexy compared with open abdominal sacrocolpopexy for vault prolapse repair: a randomised controlled trial," International Urogynecology Journal, vol. 28, pp. 1469-1479, oct 2017.

[3] R. M. Freeman, K. Pantazis, A. Thomson, J. Frappell, L. Bombieri, P. Moran, M. Slack, P. Scott, and M. Waterfield, "A randomised controlled trial of abdominal versus laparoscopic sacrocolpopexy for the treatment of post-hysterectomy vaginal vault prolapse: LAS study," International Urogynecology Journal, vol. 24, pp. 377-384, mar 2013.

[4] I. E. Nygaard, R. McCreery, L. Brubaker, A. Connolly, G. Cundiff, A. M. Weber, and H. Zyczynski, "Abdominal Sacrocolpopexy: A Comprehensive Review," Obstetrics \& Gynecology, vol. 104, pp. 805823, oct 2004.

[5] C. Maher, B. Feiner, K. Baessler, and C. Schmid, "Surgical management of pelvic organ prolapse in women," Cochrane Database of Systematic Reviews, p. 156, apr 2013.

[6] T. F. M. Vergeldt, M. Weemhoff, J. IntHout, and K. B. Kluivers, "Risk factors for pelvic organ prolapse and its recurrence: a systematic review," International Urogynecology Journal, vol. 26, pp. 1559-1573, nov 2015.

[7] R. C. Bump and P. A. Norton, "Epidemiology and natural hystory of pelvic floor dysfunction," Obstetrics and Gynecology Clinics of North America, vol. 25, pp. 723-746, dec 1998.

[8] Y. Ozog, J. Deprest, K. Haest, F. Claus, D. De Ridder, and E. Mazza, "Calculation of membrane tension in selected sections of the pelvic floor," International Urogynecology Journal, vol. 25, pp. 499-506, apr 2014.

[9] J. Deprest, L. Krofta, F. Van der Aa, A. L. Milani, J. Den Boon, F. Claerhout, and J.-P. Roovers, "The challenge of implementing laparoscopic sacrocolpopexy," International Urogynecology Journal, vol. 25, pp. 1153-1160, sep 2014.

[10] A. M. Ganatra, F. Rozet, R. Sanchez-Salas, E. Barret, M. Galiano, X. Cathelineau, and G. Vallancien, "The Current Status of Laparoscopic Sacrocolpopexy: A Review," European urology, vol. 55, no. 5, pp. 1089-1105, 2009.

[11] A. Blavier, Q. Gaudissart, G.-B. Cadière, and A.-S. Nyssen, "Comparison of learning curves and skill transfer between classical and robotic laparoscopy according to the viewing conditions: implications for training," The American journal of surgery, vol. 194, no. 1, pp. 115121, 2007.
[12] F. Claerhout, D. De Ridder, D. Van Beckevoort, G. Coremans, J. Veldman, P. Lewi, and J. Deprest, "Sacrocolpopexy using xenogenic acellular collagen in patients at increased risk for graft-related complications," Neurourology and Urodynamics, vol. 29, no. 4, pp. 563-567, 2009.

[13] R. Berguer, W. Smith, and Y. Chung, "Performing laparoscopic surgery is significantly more stressful for the surgeon than open surgery," Surgical Endoscopy, vol. 15, pp. 1204-1207, oct 2001.

[14] A. Park, G. Lee, F. J. Seagull, N. Meenaghan, and D. Dexter, "Patients Benefit While Surgeons Suffer: An Impending Epidemic," Journal of the American College of Surgeons, vol. 210, pp. 306-313, mar 2010.

[15] C. C. J. Alleblas, A. M. de Man, L. van den Haak, M. E. Vierhout, F. W. Jansen, and T. E. Nieboer, "Prevalence of Musculoskeletal Disorders Among Surgeons Performing Minimally Invasive Surgery," Annals of Surgery, vol. 266, pp. 905-920, dec 2017.

[16] F. Claerhout, J. Verguts, E. Werbrouck, J. Veldman, P. Lewi, and J. Deprest, "Analysis of the learning process for laparoscopic sacrocolpopexy: identification of challenging steps," International Urogynecology Journal, vol. 25, pp. 1185-1191, sep 2014.

[17] F. Claerhout, D. De Ridder, J. P. Roovers, H. Rommens, F. Spelzini, V. Vandenbroucke, G. Coremans, and J. Deprest, "Medium-Term Anatomic and Functional Results of Laparoscopic Sacrocolpopexy Beyond the Learning Curve," European Urology, vol. 55, pp. 14591468 , jun 2009.

[18] M. N. Thomsen and R. D. Lang, "An experimental comparison of 3-dimensional and 2-dimensional endoscopic systems in a model1 1the equipment was provided by richard wolf, knittlingen, germany.," Arthroscopy: The Journal of Arthroscopic and Related Surgery, vol. 20, no. 4, pp. $419-423,2004$.

[19] R. Guanà, L. Ferrero, S. Garofalo, A. Cerrina, D. Cussa, A. Arezzo, and J. Schleef, "Skills comparison in pediatric residents using a 2dimensional versus a 3-dimensional high-definition camera in a pediatric laparoscopic simulator," Journal of Surgical Education, vol. 74, no. 4, pp. $644-649,2017$.

[20] K. Nomura, D. Kikuchi, M. Kaise, T. Iizuka, Y. Ochiai, Y. Suzuki, Y. Fukuma, M. Tanaka, Y. Okamoto, S. Yamashita, A. Matsui, T. Mitani, and S. Hoteya, "Comparison of 3D endoscopy and conventional 2D endoscopy in gastric endoscopic submucosal dissection: an ex vivo animal study," Surgical Endoscopy, pp. 1-7, mar 2019.

[21] A. Chan, S. Chung, A. Yim, J. Lau, E. Ng, and A. Li, "Comparison of two-dimensional vs three-dimensional camera systems in laparoscopic surgery," Surgical endoscopy, vol. 11, no. 5, pp. 438-440, 1997.

[22] G. B. Hanna and A. Cuschieri, "Influence of two-dimensional and three-dimensional imaging on endoscopic bowel suturing," World journal of surgery, vol. 24, no. 4, pp. 444-449, 2000.

[23] S. Romero-Loera, L. E. Cárdenas-Lailson, F. de la Concha-Bermejillo, B. A. Crisanto-Campos, C. Valenzuela-Salazar, and M. MorenoPortillo, "Skills comparison using a 2d vs. 3d laparoscopic simulator," Cirugía y Cirujanos (English Edition), vol. 84, no. 1, pp. 37-44, 2016.

[24] M. Özsoy, P. Kallidonis, I. Kyriazis, V. Panagopoulos, M. Vasilas, G. C. Sakellaropoulos, and E. Liatsikos, "Novice surgeons: do they benefit from 3D laparoscopy?," Lasers in Medical Science, vol. 30, pp. 1325-1333, may 2015.

[25] S.-H. Kong, B.-M. Oh, H. Yoon, H. S. Ahn, H.-J. Lee, S. G. Chung, N. Shiraishi, S. Kitano, and H.-K. Yang, "Comparison of two- and three-dimensional camera systems in laparoscopic performance: a novel 3d system with one camera," Surgical Endoscopy, vol. 24, pp. 1132-1143, May 2010.

[26] Y.-K. Zeng, Z.-Y. Li, W.-L. Xuan, and J.-X. He, "Trans-oral glassesfree three-dimensional endoscopic thyroidectomy-preliminary single center experiences," Gland surgery, vol. 5, no. 6, p. 628, 2016.

[27] K. Sakamoto, T. Ohara, S. Nomura, T. Hirotomi, K. Shiwaku, and M. Hirakawa, "Glasses-free 3d display system using view control film for stereo image separation," Ferroelectrics, vol. 394, no. 1, pp. 54-67, 2010.

[28] L. Jiang, J. Liu, W. Shao, J. Li, and J. He, "Non-intubated subxiphoid uniportal video-assisted thoracoscopic thymectomy using glasses-free 3d vision," Journal of thoracic disease, vol. 8, no. 12, p. E1602, 2016.

[29] M. Li, "The world's first radical resection for lung cancer using glasses-free $3 \mathrm{~d}$ thoracoscope was completed in guangzhou," Journal of thoracic disease, vol. 7, no. 9, p. E384, 2015. 